\title{
Online Detection System of Metal Wear Debris Based on Symmetrical Magnetic Excitation Balance Magnetic Field Model
}

Kai Li ( $\sim$ likai@nuc.edu.cn )

North University of China https://orcid.org/0000-0003-3125-3919

\section{WenBin Bai}

North University of China

\section{Yuan Li}

North University of China

HaoSen Dong

North University of China

\section{RuiQing Bian}

North University of China

\section{QingQing Gong}

North University of China

YaJun Ren

North University of China

\section{Research Article}

Keywords: Fault detection, Metal wear debris, Electromagnetic induction, Symmetric magnetic excitation, Balance magnetic field

Posted Date: March 7th, 2022

DOI: https://doi.org/10.21203/rs.3.rs-1298333/v1

License: (c) (1) This work is licensed under a Creative Commons Attribution 4.0 International License. Read Full License 


\section{Title page}

\section{Online Detection System of Metal Wear Debris Based on Symmetrical Magnetic Excitation Balance Magnetic Field Model}

Kai Li, born in 1979, is currently an associate professor at School of Information and Communication Engineering, North University of China, China. His research interests include man-machine system and mechanical fault diagnosis.

Tel: +86-13753156898; E-mail: likai@ nuc.edu.cn

WenBin Bai, born in 1997, is currently a master candidate at State Key Laboratory of Fluid Power Transmission and Control, Zhejiang University, China.

E-mail: s1905009@st.nuc.edu.cn

Yuan Li, born in 1982, is currently an associate professor at School of Information and Communication Engineering, North University of China, China.

E-mail: liyuan82@nuc.edu.cn

HaoSen Dong, born in 1998, is currently a master candidate at State Key Laboratory of Fluid Power Transmission and Control, Zhejiang University, China.

E-mail: sz202105050@st.nuc.edu.cn

RuiQing Bian, born in 1998, is currently a master candidate at State Key Laboratory of Fluid Power Transmission and Control, Zhejiang University, China.

E-mail: sz202105017@st.nuc.edu.cn

QingQing Gong, born in 1996, is currently a master candidate at State Key Laboratory of Fluid Power Transmission and Control, Zhejiang University, China.

E-mail: s1905076@st.nuc.edu.cn

YaJun Ren, born in 1996, is currently a master candidate at State Key Laboratory of Fluid Power Transmission and Control, Zhejiang University, China.

E-mail: s1905036@st.nuc.edu.cn

Corresponding author: Kai Li E-mail: likai@nuc.edu.cn 


\title{
Online Detection System of Metal Wear Debris Based on Symmetrical Magnetic Excitation Balance Magnetic Field Model
}

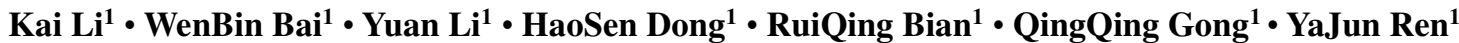

Received January xx, 201x; revised February xx, 201x; accepted March xx, 201x

(C) Chinese Mechanical Engineering Society and Springer-Verlag Berlin Heidelberg 2017

\begin{abstract}
The metal wear debris generated by wear during the operation of the mechanical equipment can reflect the fault status of the equipment, and the online monitoring of the metal wear debris in the oil can timely understand the operation information of the equipment. Aiming at the effective response of metal wear debris under symmetrical magnetic excitation, an online monitoring system for metal wear debris with dual-coil balanced magnetic excitation was developed. Based on the spatial magnetic field distribution of the current-carrying element, an analytical model of the magnetic field intensity distribution of a single-turn cur-rent-carrying ring is designed, and it is optimized as a single set of coils made of a current-carrying ring, and the coupling of the magnetic excitation of the symmetrical double coil is deduced. The magnetic field distribution model is simulated and analyzed by numerical method. The flow channel of the system is $8 \mathrm{~mm}$. After the semi-physical simulation test with the wear debris speed of 3.03-5.25 m/s, the sensor can realize the detection of $100 \mu \mathrm{m}$ ferromagnetic metal and $1000 \mu \mathrm{m}$ non-ferromagnetic metal. The experiment verifies the correctness of the simulation model of the coupled magnetic field distribution. This research provides technical support for the detection of hydraulic oil contaminants, which is of great significance for preventing hydraulic system failures and extending the working life of hydraulic equipment.
\end{abstract}

Keywords: Fault detection - Metal wear debris • Electromagnetic induction - Symmetric magnetic excitation - Balance magnetic field

Kai Li

likai@nuc.edu.cn

1 School of Information and Communication Engineering, North University of China, Taiyuan 030051 , China

\section{Introduction}

As key working fluid of mechanical equipment, hydraulic oil has the functions of transmitting hydraulic energy, lubricating friction pair of hydraulic system, cooling system and so on [1]. During the operation of mechanical equipment, wear debris formed by mechanical wear will contaminate the lubricating oil, and the polluted oil will also aggravate the wear of the moving pair and cause the failure of the mechanical equipment [2]. Wear debris are the most direct information element for revealing the tribological surface wear mechanism, monitoring the process of wear and diagnosing the type of wear failure [3]. Through the real-time detection of the type, size and concentration of pollutants, the working state of the mechanical equipment can be reflected, which is of great significance for the safety of operation and fault diagnosis of the hydraulic system [4].

The technology of oil monitoring sensor has been developed so far, mainly including optical $[5,6]$, acoustic [7], capacitive [8] and inductive [9] sensors. Optical sensors can comprehensively obtain wear debris morphology, size, color and other characteristics, but the image quality is affected by the transparency of the oil. When the lubricating oil ages and turns black, it cannot effectively image [10,11]. Acoustic sensors have high detection accuracy, but the installation location of the sensor, the selection of ultrasonic frequency, and the effects of vibration and noise will seriously affect the detection performance [12]. Capacitive sensor can detect water droplets and bubbles in oil by changing the dielectric constant, but they cannot discriminate the magnetic properties of metal wear debris in oil [13]. The inductive sensor is based on the magnetic anomaly change of the 
inspection coil caused by the scattered magnetic field of the wear debris under the magnetic excitation condition, and uses the induction signals with different trends to characterize the metal wear debris of different magnetic properties, and is not affected by the clarity of the oil [14], based on the above advantages, this kind of detection method has been highly valued in the field of metal wear debris detection. However, the environment and mechanical vibration of the sensor will affect the detection sensitivity. The demand for on-line detection and on-line fault judgment of industrial-grade mechanical equipment oil circuit wear debris has become a major technical problem.

Sensitivity and throughput of detection are two key standard indicators for measuring inductive sensors. At present, the technology to improve the detection sensitivity of the sensor mainly includes the technology of sensor structure design, the technology of circuit compensation, and the technology of signal processing related to wear debris.

The technology of sensor structure design includes the design of microfluidic sensor [15], the optimization design of sensor structure parameters [16], the design of new sensor detection structure [17], the design of sensor detection mode [18], the design of sensor internal magnetic field enhancement structure [19] and so on. With the development of microfabrication technology, the inductive detection method of oil contaminants based on microfluidic chips has been widely used, which improves the detection sensitivity of wear debris while sacrificing throughput. The uniformity of the magnetic field is determined by the structural parameters of the sensor, and the detection sensitivity of the sensor is affected by the uniformity of the magnetic field. In order to improve the detection sensitivity of wear debris, the parameters of the sensor are optimized, including the inner diameter of the coil, the width of the coil, the distance between the coils, and the number of turns of the coil [20]. According to the distribution characteristics of the magnetic field, the magnetic field generated by the coil has a limited coverage, and the inductance change caused by the wear debris passing through the weak magnetic field is weak. In order to expand the coverage of the magnetic field, the sensor probe is designed, including new sensors such as one-exciting multi-sensing and multi-exciting one-sensing, which can cover the entire oil passage section and meet the detection of wear debris at any position. In order to realize the measurement of various pollutant parameters in oil, Dalian Maritime University designed a wear debris detection device based on inductance and capacitance detection methods. By adjusting the excitation source, the inductance-capacitance mode can be switched. The measurement of various pollutants such as water droplets, air bubbles and metal wear debris with different magnetic properties in the hydraulic oil is completed [21]. The detection sensitivity of the inductive sensor is affected by the magnetization effect of ferromagnetic wear debris. The magnetization effect of ferromagnetic wear debris can be enhanced by filling paramagnetic materials [22] or adding ferrite cores [23] inside the sensor, effectively improve the detection signal-to-noise ratio of wear debris signals.

Technology of circuit compensation mainly includes resonant circuit [24], excitation coil unbalance compensation circuit [25] and so on. The coil in the parallel resonance state will amplify the coil impedance change caused by the wear debris, enhance the induced electromotive force output by the sensor, and improve the signal-to-noise ratio and sensitivity. For the three-coil sensor, the unbalance compensation circuit can effectively eliminate the unbalanced voltage caused by the asymmetry of the two excitation coils, thereby improving the detection sensitivity.

In addition to the structural design of the sensor and the technology of circuit compensation, the processing technology of the wear debris signal can also improve the detection sensitivity. The wear debris signal is a single-period sine wave signal, and the wear debris can be identified by the threshold algorithm, but this method requires a high signal-to-noise ratio. In order to improve the signal-to-noise ratio, Luo et al. [26] proposed to use the resonance-based signal decomposition to eliminate vibration and background noise. But when the interference frequency is close to the wear debris signal, its effect will be weakened. In response to this problem, Hong et al. [27] proposed an algorithm that combines band-pass filters and related algorithms, which can improve the signal-to-noise ratio by more than 2.67 times. To expand the scope of adaptation, Liu et al. [28] proposed a method based on multiple correlation windows to improve the sensitivity and adaptability of oil debris detection.

The throughput of the sensor is also an index to measure the performance of the sensor. The currently developed sensors sacrifice throughput while ensuring the detection sensitivity, which not only restricts the oil detection amount of the sensor, but also causes the inner wall of the sensor to bear greater pressure. Due to the limitation of the sensor throughput, it can only be installed in the lubricating oil bypass, so that the sensor only samples and monitors part of the lubricating oil, and the detection results are quite random. 
In innovative research on sensor structures, appropriate application of new structures can expand sensor throughput while maintaining accuracy. Zhu et al. [29] proposed a sensor structure composed of $3 \times 3$ sensing channels, which can realize the simultaneous detection of 9 sensor signals, and the sensitivity of the sensor to detect ferromagnetic wear debris can reach $50 \mu \mathrm{m}$ at an aperture of $1 \mathrm{~mm}$. Based on this, $\mathrm{Wu}$ et al. [30] proposed a multi-channel wear debris detection method based on time division multiplexing, which improved the throughput and ensured the detection accuracy in the case of using only one excitation signal to excite multiple sensing coils. In order to improve the flux of the sensor, Feng et al. [31,32] proposed a high-throughput sensor driven by constant current, which can form a local high-gradient magnetic field, Iron wear debris of $20 \mu \mathrm{m}$ can be detected in the oil circuit with a diameter of $1.5 \mathrm{~mm}$. However, the detection area of this sensor is limited by the radial height, which is not enough to characterize the distribution of wear debris in the lubricating oil system. Based on this foundation, Xiao et al. [33] designed a new type of six-excitation structure inductive sensor to cover the $40 \mathrm{~mm}$ diameter flow channel, but the magnetic field coverage area of the sensor is still limited. Ren et al. [34] proposed an inductive wear debris monitoring sensor with a "single impulse and multiple senses", which can detect iron wear debris of 120 $\mu \mathrm{m}$ and copper wear debris of $210 \mu \mathrm{m}$ in a pipe with an inner diameter of $34 \mathrm{~mm}$. However, this structure divides the flow channel into multiple regions for detection, which will increase the oil flow resistance and affect the operation of mechanical equipment.

In order to achieve high-sensitivity detection under high-throughput conditions of inductive wear debris monitoring sensors. This paper takes the double-coil excitation method as the research object of the metal wear debris test. Aiming at the effective response of metal wear debris under the symmetrical magnetic excitation, based on the spatial magnetic field distribution model of the current-carrying element, the coupled magnetic field of the symmetrical double-coil magnetic excitation is established. The distribution model is analyzed, and its distribution characteristics are analyzed to simulate the magnetic field distribution of the sensor, which provides a theoretical basis for the actual sensor design. It is verified by semi-simulation experiments that the flow channel is $8 \mathrm{~mm}$ and the wear debris velocity is $3.03-5.52 \mathrm{~m} / \mathrm{s}$. The sensor can detect $100 \mu \mathrm{m}$ ferromagnetic metals and $1000 \mu \mathrm{m}$ non-ferromagnetic metals.

\section{Theory of magnetic field distribution in detection area}

\subsection{Symmetrical magnetic excitation balance magnetic field model}

When the sensor is in operation, the metal wear debris are enveloped in the oil and pass through the sensor, the signal output by the metal wear debris disturb the magnetic field is affected by the internal magnetic field of the sensor. In order to analyze the relationship between the output signal of sensor and the internal magnetic field, a schematic diagram of the internal magnetic field distribution of the sensor is established.

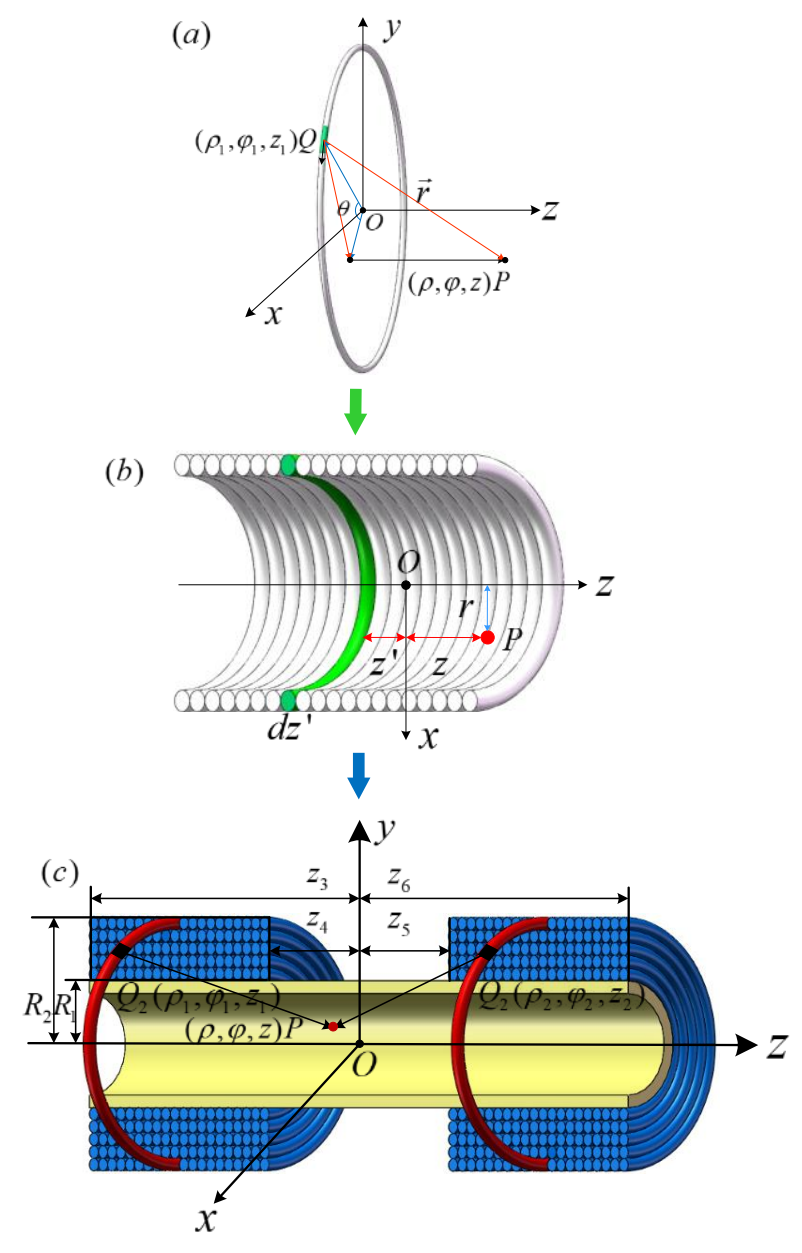

Figure 1 Schematic diagram of the magnetic field distribution inside the sensor.

In the single-turn current-carrying circular coil space with excitation radius $\rho$ and excitation current $I$, the magnetic field distribution at arbitrary point is shown in Figure 1 (a). The schematic diagram uses a cylindrical coordinate system as the representation method. Taking the 
circular surface where the current-carrying circular coil is located as the Oxy plane, the center of the coil is the coordinate origin $O$, the axis direction of the coil is the $z$ axis, the coordinate of the current-carrying element of the coil is $Q\left(\rho_{1}, \varphi_{1}, z_{1}\right)$, point $P(\rho, \varphi, z)$ is arbitrarily selected inside coil. $\vec{r}$ is the position vector between the current element and point $P, \mu_{0}$ is vacuum permeability.

The magnetic induction at any point $P$ in a single-turn current-carrying coil is:

$$
\left\{\begin{array}{c}
d B=\int_{0}^{2 \pi} \frac{\mu_{0} I}{4 \pi} \frac{d \vec{l} \times \vec{r}}{r^{3}} d \theta \\
d \vec{l}=\left(-\rho_{1} \sin \varphi_{1} \vec{i}+\rho_{1} \cos \varphi_{1} \vec{j}\right) d \varphi_{1} \\
r=\sqrt{\rho^{2}+\rho_{1}^{2}-2 \rho_{1} \rho \cos \left(\varphi-\varphi_{1}\right)+z^{2}} \\
\vec{r}=\left(\rho \cos \varphi-\rho_{1} \cos \varphi_{1}\right) \vec{i}+\left(\rho \sin \varphi-\rho_{1} \sin \varphi_{1}\right) \vec{j}+z \vec{k}
\end{array}\right.
$$

The schematic diagram of the internal magnetic field distribution of a single set of excitation coils is shown in Figure 1 (b). A single set of excitation coils is densely wound with multiple turns of coils. Each turn of the coil can be approximated as a closed-loop circular current. $n$ is the number of turns per unit length. The magnetic induction intensity at any point $P$ in a single set of excitation coils is formed by the superposition of the magnetic induction intensity excited by $n$ turns of the current-carrying circular coil at this point. A current-carrying circular coil with $n d z^{\prime}$ turns is equivalent to a single-turn current-carrying coil with a current of Indz'. $r$ is the vertical distance from point $P$ to the axis of the solenoid, and $z$ is the distance from the projection point of $P$ on the $\mathrm{z}$ axis to the origin $O$.

The magnetic induction at any point $P$ in a single set of excitation coils is:

$$
\left\{\begin{array}{c}
B(\rho, z)=\frac{\mu_{0} n I \rho_{1}}{4 \pi} \int_{-l / 2}^{l / 2} \int_{0}^{2 \pi} \frac{d \vec{l} \times \vec{r}}{r^{3}} d \theta d z^{\prime} \\
d \vec{l}=\left(-\rho_{1} \sin \varphi_{1} \vec{i}+\rho_{1} \cos \varphi_{1} \vec{j}\right) d \varphi_{1} \\
r=\sqrt{\rho^{2}+\rho_{1}^{2}-2 \rho_{1} \rho \cos \left(\varphi-\varphi_{1}\right)+\left(z-z^{\prime}\right)^{2}} \\
\vec{r}=\left(\rho \cos \varphi-\rho_{1} \cos \varphi_{1}\right) \vec{i}+\left(\rho \sin \varphi-\rho_{1} \sin \varphi_{1}\right) \vec{j}+\left(z-z^{\prime}\right) \vec{k}
\end{array}\right.
$$

The internal magnetic field distribution of the symmetrical excitation coil is shown in Figure 1 (c). the center of the coil is the coordinate origin $O$, the axis direction of the coil is the $z$ axis. The coordinates of the current-carrying elements of the two excitation coils are $Q_{1}\left(\rho_{1}, \varphi_{1}, z_{1}\right) 、 Q_{2}\left(\rho_{2}, \varphi_{2}, z_{2}\right)$. Take any point $P(\rho, \varphi, z)$ inside the coil, $z_{3} 、 z_{4} 、 z_{5} 、 z_{6}$ are the axial coordinates of the front and rear ends of the two sets of excitation coils. $n$ is windings per unit length, $R_{1}, R_{2}$ are the inner and outer radius of the solenoid, respectively, $\vec{r}$ is the position vector between the current element and point $P$, $\mu_{0}$ is vacuum permeability.

The magnetic field strength of the left single set of excitation coils at point $P$ is:

$$
\left\{\begin{array}{c}
\vec{B}_{1}=\int_{R_{1}}^{R_{2}} \int_{z_{3}}^{z_{4}} \int_{0}^{2 \pi} \frac{n \mu_{0} I}{4 \pi} \frac{d \overrightarrow{l_{1}} \times \vec{r}_{1}}{r_{1}^{3}} d \theta_{1} d z_{1} d \rho_{1} \\
d \vec{l}_{1}=\left(-\rho_{1} \sin \varphi_{1} \vec{i}+\rho_{1} \cos \varphi_{1} \vec{j}\right) d \varphi_{1} \\
r_{1}=\sqrt{\rho^{2}+\rho_{1}^{2}-2 \rho_{1} \rho \cos \left(\varphi-\varphi_{1}\right)+\left(z-z_{1}\right)^{2}} \\
\vec{r}_{1}=\left(\rho \cos \varphi-\rho_{1} \cos \varphi_{1}\right) \vec{i}+\left(\rho \sin \varphi-\rho_{1} \sin \varphi_{1}\right) \vec{j}+\left(z-z_{1}\right) \vec{k}
\end{array}\right.
$$

The sensor structure used in this system is a symmetrical double-coil structure. The intensity of the magnetic field at arbitrary point $P$ inside the sensor is simultaneously affected by the excitation coils on both sides. Analyzing the internal magnetic field distribution of a single excitation coil cannot completely represent the actual magnetic field distribution inside the sensor. Therefore, based on the mathematical model of the internal magnetic field distribution of the single coil, the internal magnetic field of the single set of excitation coils on the right is theoretically analyzed and calculated.

The magnetic field strength of the right excitation coil at point $P$ is:

$$
\left\{\begin{array}{c}
\overrightarrow{B_{2}}=\int_{R_{1}}^{R_{2}} \int_{z_{5}}^{z_{6}} \int_{0}^{2 \pi}-\frac{n \mu_{0} I}{4 \pi} \frac{d \overrightarrow{l_{2}} \times \vec{r}_{2}}{r_{2}^{3}} d \theta_{2} d z_{2} d \rho_{2} \\
d \overrightarrow{l_{2}}=\left(-\rho_{2} \sin \varphi_{2} \vec{i}+\rho_{2} \cos \varphi_{2} \vec{j}\right) d \varphi_{2} \\
r_{2}=\sqrt{\rho^{2}+\rho_{2}^{2}-2 \rho_{2} \rho \cos \left(\varphi-\varphi_{2}\right)+\left(z-z_{2}\right)^{2}} \\
\overrightarrow{r_{2}}=\left(\rho \cos \varphi-\rho_{2} \cos \varphi_{2}\right) \vec{i}+\left(\rho \sin \varphi-\rho_{2} \sin \varphi_{2}\right) \vec{j}+\left(z-z_{2}\right) \vec{k}
\end{array}\right.
$$

Combing with the mathematical model of the magnetic field strength of the two excitation coils at point $P$, the radial magnetic induction intensity of the symmetrical excitation coil at any reference point $P$ can be obtained as:

$$
\begin{aligned}
B_{r}(\rho, z)= & \frac{\mu_{0} n I}{4 \pi}\left[\int_{R_{1}}^{R_{2}} \int_{z_{3}}^{z_{4}} \int_{0}^{2 \pi} \frac{\rho_{1}\left(z-z_{1}\right) \cos \theta_{1}}{r_{1}^{3}} d \theta_{1} d z_{1} d \rho_{1}-\right. \\
& \left.\int_{R_{1}}^{R_{2}} \int_{z_{5}}^{z_{6}} \int_{0}^{2 \pi} \frac{\rho_{2}\left(z-z_{2}\right) \cos \theta_{2}}{r_{2}^{3}} d \theta_{2} d z_{2} d \rho_{2}\right]
\end{aligned}
$$

The radial magnetic induction intensity of the symmetrical excitation coil at any reference point $P$ is: 


$$
\begin{aligned}
B_{z}(\rho, z)= & \frac{\mu_{0} n I}{4 \pi}\left[\int_{R_{1}}^{R_{2}} \int_{z_{3}}^{z_{4}} \int_{0}^{2 \pi} \frac{\rho_{1}\left(\rho_{1}-\rho \cos \theta_{1}\right)}{r_{1}^{3}} d \theta_{1} d z_{1} d \rho_{1}-\right. \\
& \left.-\int_{R_{1}}^{R_{2} z_{5}} \int_{z_{5}}^{2 \pi} \int_{0}^{2 \pi} \frac{\rho_{2}\left(\rho_{2}-\rho \cos \theta_{2}\right)}{r_{2}^{3}} d \theta_{2} d z_{2} d \rho_{2}\right]
\end{aligned}
$$

This mathematical model can represent the vector sum of the magnetic field intensity of two excitation coils acting at arbitrary point in the solenoid at the same time, restore the magnetic field distribution inside the sensor under ideal conditions, and provide a theoretical basis for analyzing the relationship between the sensor signal output and the internal magnetic field.

\subsection{Model simulation}

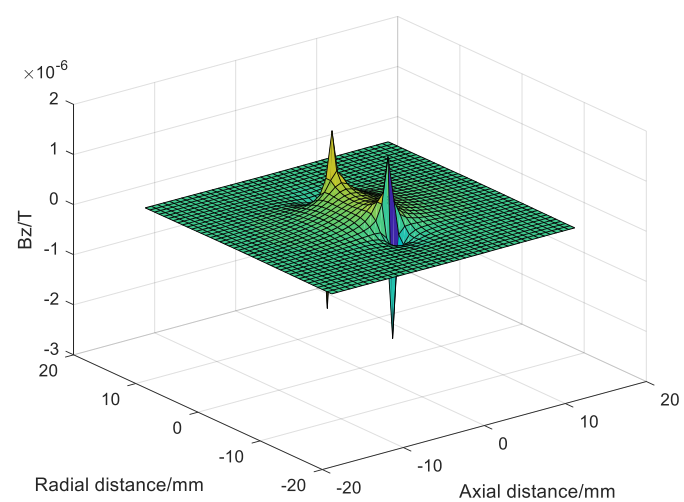

Figure $2 \mathrm{Bz}$ of a single-turn current-carrying ring.

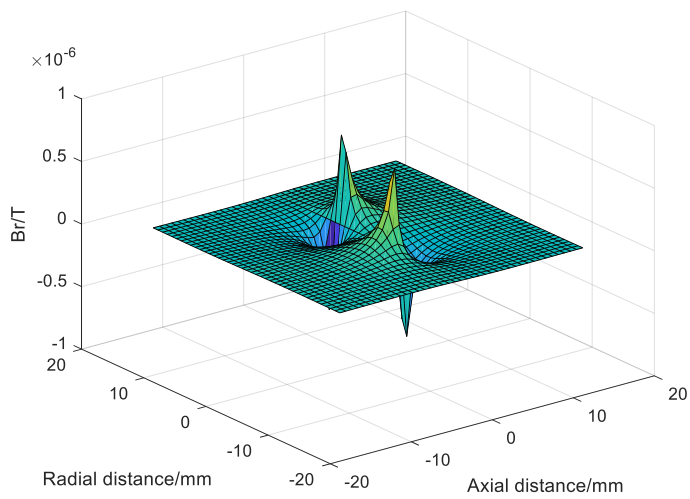

Figure $3 \mathrm{Br}$ of a single-turn current-carrying ring.

The axial and radial magnetic field distributions of a single-turn current-carrying ring are shown in Figure 2 and Figure 3. It can be seen from Figure 2 that $\mathrm{Bz}$ is distributed in a saddle shape, and is symmetrically distributed with the axial position of 0 as the axis of symmetry. In the axial direction of the current-carrying ring, from the outside of the ring to the position as 0 in the axial direction, the magnetic field intensity gradually increases to the peak value. In the radial direction of the ring, from the center of the ring to the inner wall of the ring, the magnetic field strength gradually increases to the peak value, and reaches the maximum value on the inner wall of the ring. Br takes the axial position of 0 as the rotation axis and is symmetrically distributed in the center, and the magnetic field intensity reaches the maximum value on the inner wall of the ring.

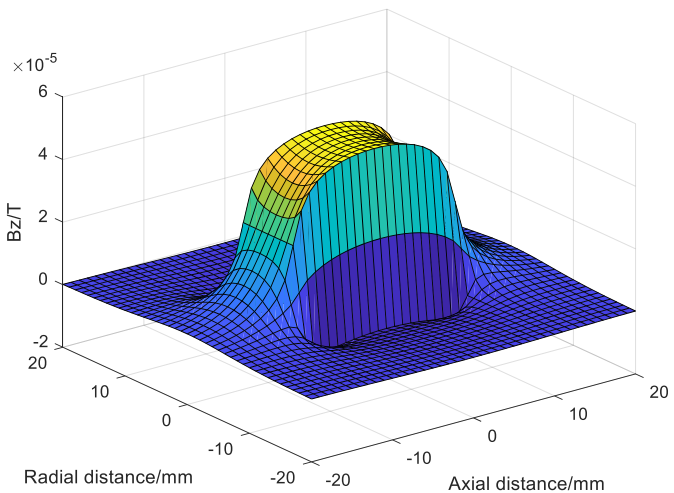

Figure $4 \mathrm{Bz}$ of a single set of coils.

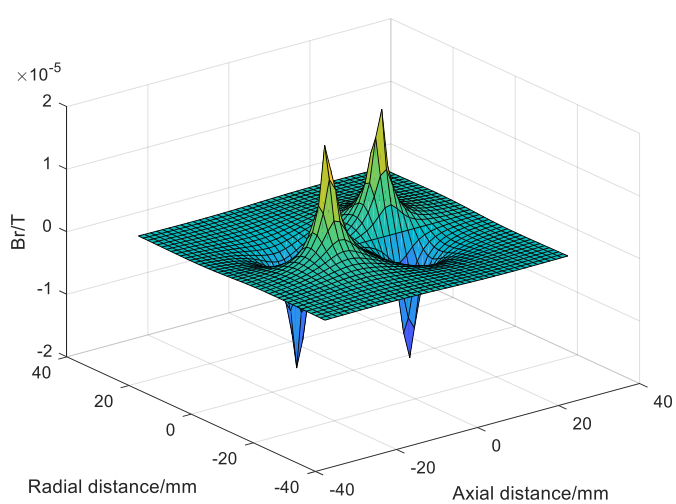

Figure $5 \mathrm{Br}$ of a single set of coils.

The axial and radial magnetic field distributions of a single set of coils are shown in Figure 4 and Figure 5. It can be seen from Figure 4 that $\mathrm{Bz}$ is distributed in a saddle shape, and is symmetrically distributed with the axial position of 0 as the axis of symmetry. In the axial direction of the coil, from the outside of the coil to the axial position of 0 , the magnetic field strength gradually increases to the peak value. In the radial direction of the coil, from the center of the coil to the inner wall of the coil, the magnetic 
field strength gradually increases to the peak value, and reaches the maximum value on the inner wall of the coil. $\mathrm{Br}$ takes the axial position of 0 as the rotation axis and is symmetrically distributed in the center, and the magnetic field intensity reaches the maximum value on the inner wall of the coil.

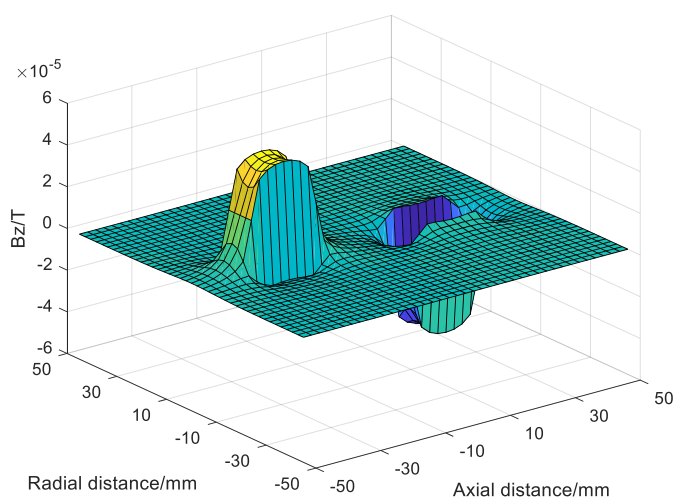

Figure $6 \mathrm{Bz}$ of the symmetrical excitation coils.

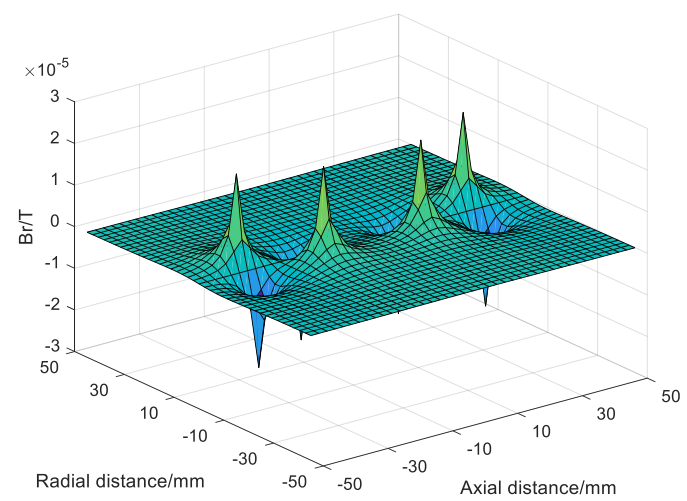

Figure $7 \mathrm{Br}$ of the symmetrical excitation coils.

The axial and radial magnetic field distributions of the symmetrical excitation coils are shown in Figure 6 and Figure 7. The magnetic field distribution of the single-sided coil in the picture is the same as that of a single set of coils. It can be seen from Figure 6 that the single set of coils $\mathrm{Bz}$ is still distributed in a saddle shape. Because the two excitation coils are wound in opposite directions, $\mathrm{Bz}$ is distributed symmetrically around the center of the rotation axis at the axial distance of 0 . When the axial distance is 0 , the magnetic field component $\mathrm{Bz}$ is 0 , and $\mathrm{Bz}$ reaches a peak value at the center of the two excitation coils. In the radial direction of the coil, from the center of the coil to the inner wall, the magnetic field strength gradually increases to a peak value, and the magnetic field strength reaches the maximum value at the inner wall of the coil. It can be seen from Figure 7 that $\mathrm{Br}$ is symmetrically distributed with the axial distance of 0 as the symmetry axis. The closer to the inner wall of the sensor, the greater the magnetic induction intensity, which conforms to the distribution trend of the actual magnetic field and verifies the reliability of the model.

\section{Schematic diagram of the structural model of the central weakly perturbed magnetic excitation detection}

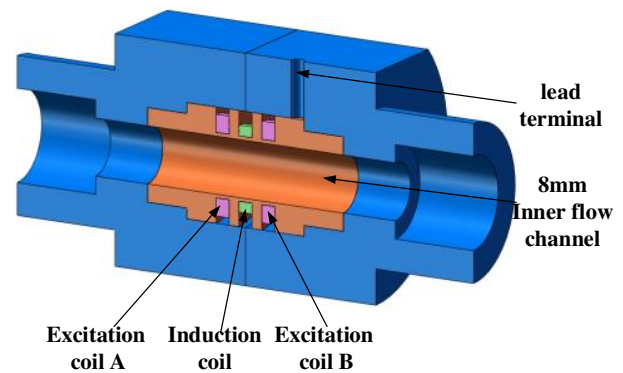

Figure 8 Schematic diagram of the central weakly disturbed magnetic excitation detection structure.

The size range of metal wear debris generated by friction of mechanical equipment such as gear boxes is in the micrometer range, and the coupled induced electromotive force generated by the magnetic field disturbed by metal wear debris are weak, which increases the difficulty of detection. The model of magnetic excitation detection structure with central weak disturbance designed to improve the detection sensitivity of wear debris is shown in Figure 8. The designed symmetrical dual-coil sensor uses high-quality enameled wire to wind three sets of coils side by side on a tubular skeleton. The winding direction of the excitation coils at both ends of the sensor is determined by the excitation method of the excitation signal source. The excitation coils using the same excitation signal source are wound in opposite directions. Conversely, the excitation coils of the excitation signal source with a phase difference of $180^{\circ}$ are wound in the same direction. When the excitation magnetic field formed by the sensor matching the above excitation and winding methods is opposite, the magnetic field intensity at the midpoint of the interval between the two excitation coils can theoretically be zero. The induction coil is located at the center of the excitation coils at both ends and the three groups of induction coils are separated by the skeleton. When the induction coil is in a zero magnetic field environment, the induced electromotive force output by the induction coil is 
zero. When the sensor is installed in the lubricating oil, the lubricating oil carries the wear debris through the sensor, the wear debris disturb the zero magnetic field state of the center, the sensor captures the change of the inductance when the wear debris pass through the coil, and the change of the inductance is converted into the change of the induced electromotive force. The magnitude and phase of the induced electromotive force are detected to determine the size and properties of the metal abrasive particles passing through the sensor.

\section{Detection system}

\subsection{Design of detection system}

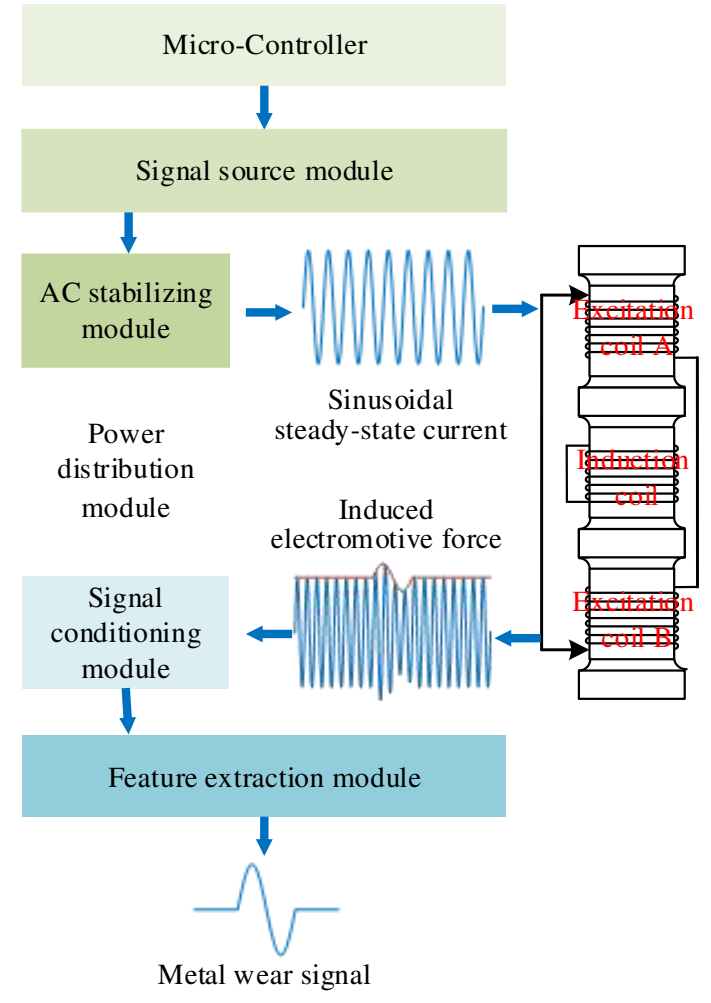

Figure 9 Block diagram of system.

The block diagram of system is shown in Figure 9, including an inductive differential metal wear debris detection sensor, a signal source module, an AC (alternating current) stabilizing module, a signal conditioning module, a signal feature extraction module and a power distribution module. The power distribution module converts the input $24 \mathrm{~V}$ DC power into multiple types of direct current stable power output to meet the different voltage and power requirements of each functional module. The excitation system of the metal wear debris sensor is composed of a signal source module and an AC stabilizing module. The sine wave signal generated by the signal source module passes through the $\mathrm{AC}$ stabilizing module and outputs a sinusoidal steady current excitation current proportional to the sinusoidal waveform voltage. The excitation current is input to the excitation coil to generate a dynamic alternating magnetic field with opposite polarity. The signal source module needs to output an excitation signal with the same phase as the sinusoidal signal to the signal conditioning module as the reference frequency signal of the demodulation module. The signal conditioning module is responsible for amplifying and detecting the weak signal output by the sensor coil. The signal feature extraction module includes wear debris magnetic discrimination, number estimation, and size classification.

\subsection{System of circuits}

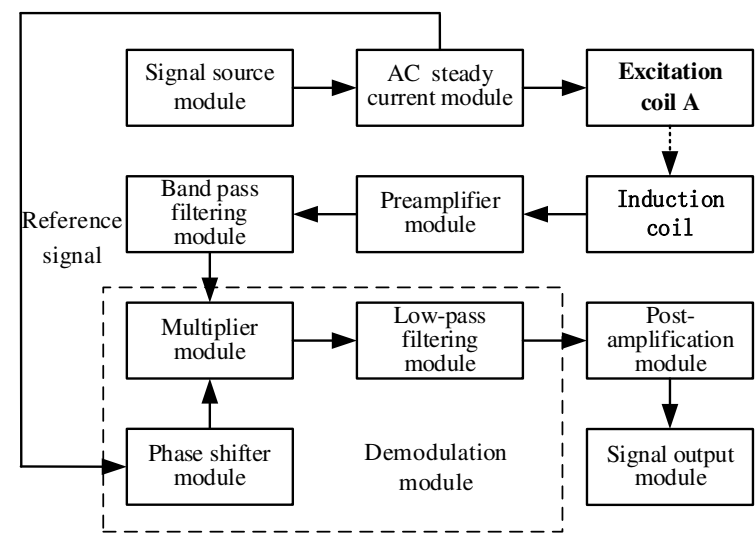

Figure 10 Block diagram for circuit system design.

The block diagram of the circuit system design is shown in Figure 10. The excitation system of the sensor is composed of a signal source module and AC stabilizing module. The sine wave signal with pure spectrum generated by the signal source module will output a sine steady current excitation current proportional to the sine waveform voltage after passing through the AC stabilizing module. A sinusoidal steady-state excitation current is input to the excitation coil to generate an excitation magnetic field. The excitation magnetic fields cancel each other at the induction coil and form a zero magnetic field environment, and the induced electromotive force generated after the wear debris disturb the magnetic field is connected to the signal conditioning module in the circuit system. The signal conditioning module includes pre-amplification, band-pass filtering, demodulation, low-pass filtering and post-amplification circuits. The induction signal generated by the magnetic field of the wear debris disturbance is weak and cannot meet the 
monitoring requirements. The signal-to-noise ratio of the wear particle signal is improved after the pre-amplification and band-pass filter module. Aiming at the characteristics of the amplitude modulation signal of the wear debris signal, a demodulation circuit is designed, which multiplies the reference signal and the induction signal, and then extracts the wear debris signal in the amplitude modulation signal through the low-pass filter module, completes the signal demodulation, and analyzes the characteristics of the wear debris. The signal is amplified again to facilitate processing by the signal feature extraction module.

\section{Experiment}

\subsection{Testing platform}

The simulation and analysis of the magnetic field distribution inside the sensor demonstrate the feasibility of the sensor. In order to prove the correctness of the simulation results and verify the actual detection effect of the wear debris sensor, an experimental platform of the wear debris detection system is built, and the corresponding experimental scheme is designed. The experimental platform includes an excitation and detection unit, a three-coil inductive sensor, a regulated power supply, and a host computer. The sensor monitors the magnetism and size of the wear debris in the lubricating oil, and the excitation unit provides the sensor with a sinusoidal steady-state excitation current to generate a dynamic alternating magnetic field. Aiming at the problem of low signal-to-noise ratio of the induced signal output by the sensor due to interference such as environmental noise, the detection unit performs processing such as signal conditioning and feature extraction, which enhances the signal-to-noise ratio of the induced signal and improves the sensor's ability to distinguish tiny metal wear debris, the regulated power supply provides stable voltage for the system, and the host computer filters and displays the obtained data.
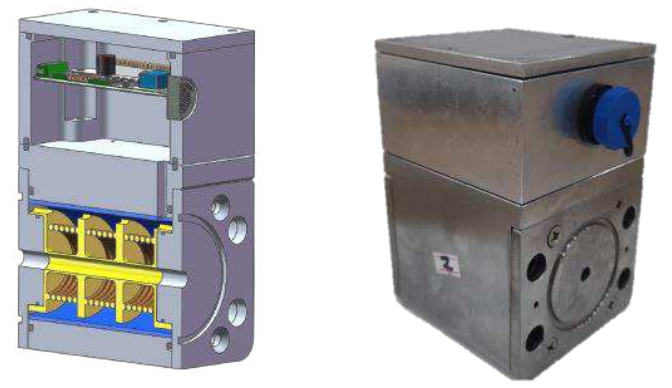

Figure 11 Wear debris sensor.
During the operation of the mechanical equipment, the friction of the mechanical equipment of the composite material leads to the existence of ferromagnetic and diamagnetic metal wear debris in the oil. The sensor mainly detects the above-mentioned different magnetic wear debris. The sample wear debris prepared in the experiment is represented by iron as a ferromagnetic metal and copper as a diamagnetic metal. The vernier caliper is used to calibrate the metal wear debris, and the diameters of iron wear debris are $100 \mu \mathrm{m} 、 300 \mu \mathrm{m} 、 1000 \mu \mathrm{m}$. The diameters of copper wear debris are $1000 \mu \mathrm{m} 、 1300 \mu \mathrm{m}$. $1500 \mu \mathrm{m}$. Metal wear debris of different sizes are encapsulated in a heat shrinkable tube and stored as samples for metal wear debris calibration test. The prepared sample wear debris are shown in Figure 12.

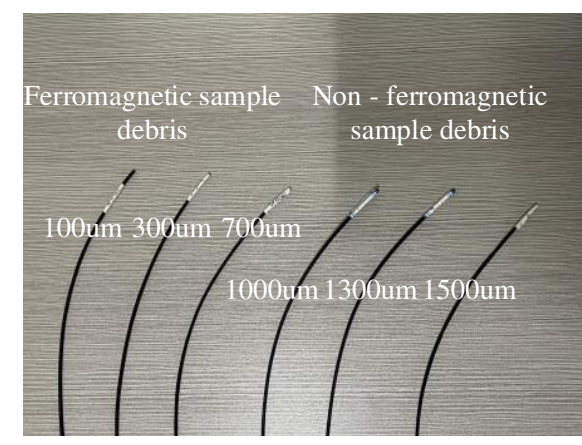

Figure 12 Wear debris of different sizes.

The three-coil inductive sensor designed in the experiment is wound with high-quality enameled wire. The parameters of the coil parameters include coil turns, coil length and so on. The output signal of the sensor is affected by the number of turns of the coil. If the number of turns of the excitation coil is too much, the impedance of the excitation coil will increase, and the excitation power needs to be increased to drive the magnetic field. If the number of turns is too small, the dynamic magnetic field strength generated by the excitation coil is weak, which is not conducive to the magnetization of wear debris. If the number of turns of the induction coil is too much, the impedance of the induction coil is too high, and an additional impedance matching circuit needs to be designed. If the number of turns is too small, the induction signal is weak, which is not conducive to extracting the wear debris signal from the complex environment. The two excitation coils in the sensor designed in the experiment have opposite winding specifications and the same size. The inner diameter of the oil pipeline is $8 \mathrm{~mm}$, the number of turns is 300 turns, and the axial length is $20 \mathrm{~mm}$. The 
number of turns of the induction coil is 1500 and the axial length is $20 \mathrm{~mm}$.

In order to verify the detection effect of the sensor on wear debris, $100 \mu \mathrm{m} 、 300 \mu \mathrm{m} 、 1000 \mu \mathrm{m}$ iron debris, 1000 $\mu \mathrm{m} 、 1300 \mu \mathrm{m} 、 1500 \mu \mathrm{m}$ copper wear debris shall be numbered (1) - (6) respectively. According to the numbering sequence, 5 groups of experiments shall be carried out for each number.

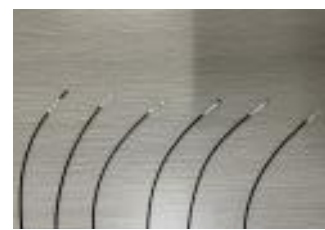

The calibration of the sample debris

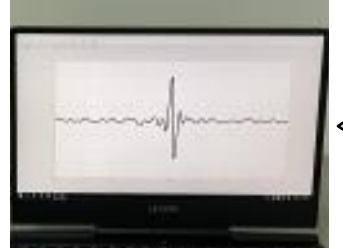

Detection unit

Display of data
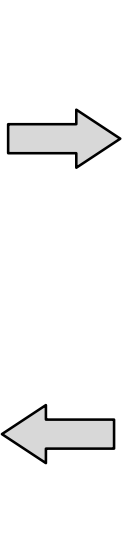

The placement of sample debris

Wear debris pass the sensor

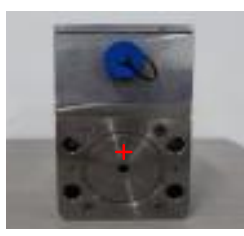

The setting of default direction

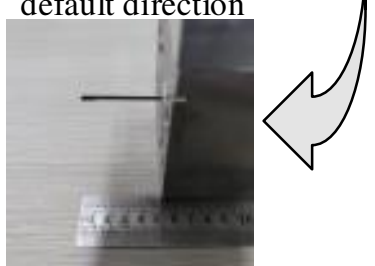

Figure 13 Experimental process.

During the operation of the mechanical equipment, the structure of the mechanical equipment determines the flow direction and flow rate of the oil. When placing the sensor, it is necessary to consider the changing trend of the wear debris sensing signal. Theoretically, when the sample wear debris pass through the sensor, the induced signal of the wear debris is a sine wave-like curve. When the wear debris are ferromagnetic wear debris, the induction signal increases first and then decreases. When the wear debris are diamagnetic wear debris, the variation trend of induction signal is opposite to that of iron wear debris, and the induction signal decreases first and then increases. At this time, the direction of the inflow of the sample wear debris into the sensor is set as the default passing direction of the wear debris, and marked on the sensor housing.

The magnetization and eddy current effect of the wear debris are mainly affected by the magnetic field in the axial and radial directions inside the sensor. When the hand-held wear debris pass through the sensor, the magnetic field of the human body will affect the axial magnetic field distribution of the sensor and interfere with the detection of the wear debris by the sensor. During the actual operation of the mechanical equipment, the sensor is placed inside the mechanical equipment, and the human body is far away from the sensor. In order to restore the actual operation of the mechanical equipment, the human body should be kept away from the sensor. In the experiment, a non-elastic nylon rope was used to tie the heat-shrinkable tube carrying the sample wear debris through the sensor.

In actual working conditions, the sensor is installed in the mechanical equipment, and the oil passes through the sensor with metal wear debris. The oil will not affect the magnetic field distribution inside the sensor due to its material properties. In this experiment, the experimental device is simplified according to the characteristics of oil, and the semi-physical simulation experiment is carried out.

During the experiment, the test is carried out in the order of numbers (1)-(6). After completing the preparation operation, make the sample wear debris flow in according to the default direction set by the sensor, and flow out from the other direction of the sensor. When the inductive signal of the wear debris passing through the sensor is transmitted to the upper computer and displayed, a test of the sample wear debris is completed.

\subsection{Test of sensor detection sensitivity}

The semi-physical simulation experiments were carried out using ferromagnetic sample debris and non-ferromagnetic sample debris of different sizes prepared in the laboratory. The output signal of ferromagnetic sample debris and non-ferromagnetic sample debris passing through the sensor is shown in Figure 14 and Figure 15.

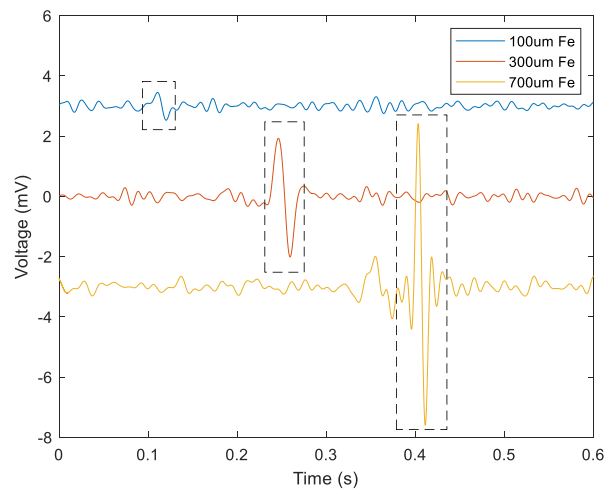

Figure 14 Iron wear debris output signal.

When the metal wear debris pass through the dynamic alternating magnetic field, an eddy current will be formed inside the metal wear debris. According to Lenz's law, the magnetic flux generated by the eddy current will resist the change of the original magnetic flux, and the magnetic medium will also have a magnetization effect in the 
magnetic field, which will cause changes in the magnetic flux density around the periphery.

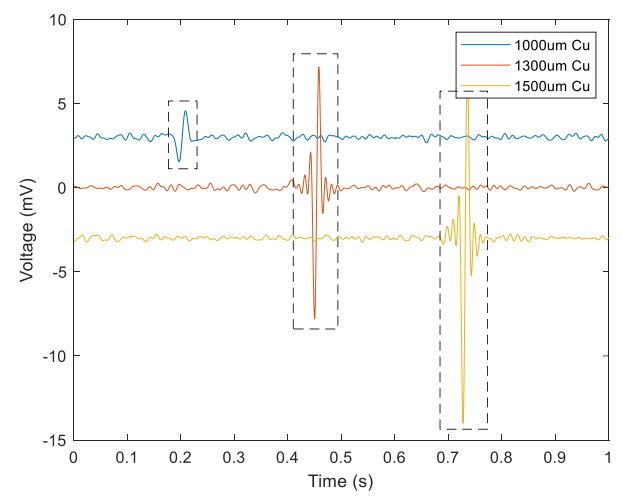

Figure 15 Copper wear debris output signal.

The experimental results of iron wear debris show that when ferromagnetic metal wear debris pass through the dynamic alternating magnetic field generated by the sensor, due to the paramagnetic properties of the material, the magnetization effect of the wear debris is much greater than the eddy current effect, and the induced signal will first increase. When the paramagnetic metal wear debris flow from the inside of the sensor to the outside, the induced signal will decrease according to Lenz's law.

The experimental results of copper wear debris show that when non-ferromagnetic metal wear debris pass through the dynamic alternating magnetic field generated by the sensor from the outside, the eddy current effect is greater than the magnetization effect, and the induced signal will decrease. When it flows to the outside, the induction signal will increase according to Lenz's law.

Table 1 Sample wear debris output signal.

\begin{tabular}{ccccc}
\hline S.N. & Material & $\begin{array}{c}\text { Grain diameter } \\
d / \mu m\end{array}$ & $\begin{array}{c}\text { Peak-to-peak } \\
\text { value } \\
V_{p p} / m V\end{array}$ & $\begin{array}{c}\text { Velocity } \\
v / m \cdot s^{-1}\end{array}$ \\
\hline 1 & Iron & 100 & 0.92 & 3.76 \\
2 & Iron & 300 & 3.98 & 3.03 \\
3 & Iron & 700 & 10.01 & 5.25 \\
4 & Copper & 1000 & 3.01 & 3.07 \\
5 & Copper & 1300 & 14.97 & 5.25 \\
6 & Copper & 1500 & 21.19 & 4.95 \\
\hline
\end{tabular}

Table 1 summarizes the signal characteristics of different sizes of metal wear debris when they pass through the sensor. The specific performance is the peak-to-peak voltage amplitude and velocity of the metal wear debris. The experimental results show that under the oil path experiment with aperture of $8 \mathrm{~mm}$ and the highest flow rate of $5.25 \mathrm{~m} / \mathrm{s}$, the system can detect the smallest ferromagnetic debris grain diameter of $100 \mu \mathrm{m}$ and the smallest non-ferromagnetic debris grain diameter of 1000 $\mu \mathrm{m}$.

\section{Conclusions}

In this paper, a metal wear debris online monitoring sensor with symmetrical double-coil balanced magnetic excitation is designed, and a coupled magnetic field distribution model of symmetrical double-coil magnetic excitation is established. The sensor avoids the influence of the primary magnetic field on the response magnetic field of the metal wear debris by using the symmetrical characteristic of the excitation magnetic field region where the detection coil is located. Through the oil circuit experiment with a diameter of $8 \mathrm{~mm}$, the effective detection of ferromagnetic wear debris with a particle size of $100 \mu \mathrm{m}$ and non-ferromagnetic wear debris with a particle size of 1000 $\mu \mathrm{m}$ is realized. The research in this paper provides technical support for the rapid differentiation and detection of hydraulic oil pollutants, which can achieve the purpose of failure analysis of hydraulic systems and prolonging the working life of hydraulic equipment.

\section{Declaration}

\section{Acknowledgements}

Not applicable.

\section{Availability of data and materials}

The datasets supporting the conclusions of this article are included within the article.

\section{Funding}

Supported by National Natural Science Foundation of Shanxi Province, China (Grant No. 201901D211251, 20210302123058).

\section{Authors' contributions}

The author' contributions are as follows: Kai Li was in charge of the whole trial; WenBin Bai wrote the manuscript; Yuan Li analyzed the data; HaoShen Dong carried out data simulation; RuiQing Bian, QingQing Gong, and YaJun Ren assisted with sampling and laboratory analyses.

\section{Competing interests}

The authors declare no competing financial interests. 


\section{References}

[1] Zhu X L, Zhong C, and Zhe J. Lubricating oil conditioning sensors for online machine health monitoring - a review. Tribology International, 2017, 109: 473-484

[2] Sun J Y, Wang L M, Li J F, et al. Online oil debris monitoring of rotating machinery: A detailed review of more than three decades. Mechanical Systems and Signal Processing, 2021, 149: 107341

[3] Du L, Zhu X, Han Y, et al. High throughput wear debris detection in lubricants using a resonance frequency division multiplexed sensor. Tribology Letters, 2013, 51: 453-460

[4] Li B, Wu W, Zhou M, et al. A full field-of-view online visual ferrograph debris detector based on reflected light microscopic imaging. IEEE Sensors Journal, 2021, 21: 16584-16597

[5] Haiden C, Wopelka T, Jech M, et al. A microfluidic chip and dark-field imaging system for size measurement of metal wear particles in oil. IEEE Sensors Journal, 2016, 16: 1182-1189

[6] Feng S, Qiu G, Luo J, et al. A wear debris segmentation method for direct reflection online visual ferrography. Sensors (Basel), 2019, 19: 723.

[7] Johansen R, Dupont S, Christiansen U N, et al. An in-line, high-flowrate, and maintenance free ultrasonic sensor with a high dynamic range for particle monitoring in fluids. IEEE Sensors Journal, 2018, 18: 2299-2304

[8] Han Z B, Wang Y S, and Qing X L. Characteristics study of in-situ capacitive sensor for monitoring lubrication oil debris. Sensors (Basel), 2017, 17:2851.

[9] Wu S, Liu Z J, Yuan H C, et al. Multichannel inductive sensor based on phase division multiplexing for wear debris detection. Micromachines (Basel), 2019, 10: 246.

[10]Peng Y P, Cai J H, Wu T H, et al. Online wear characterisation of rolling element bearing using wear particle morphological features. Wear, 2019, 430-431: 369-375

[11]Cao W, Dong G N, Xie Y B, et al. Prediction of wear trend of engines via on-line wear debris monitoring. Tribology International, 2018, 120: 510-519

[12] Xu C, Zhang P L, Wang H G, et al. Ultrasonic echo waveshape features extraction based on qpso-matching pursuit for online wear debris discrimination. Mechanical Systems and Signal Processing, 2015, 60-61: 301-315

[13]Sun Y S, Jia L C, and Zeng Z M. Hyper-heuristic capacitance array method for multi-metal wear debris detection. Sensors (Basel), 2019, 19: 515 .

[14]Yoon J S, Park J, Ahn H R, et al. Microfluidic airborne metal particle sensor using oil microcirculation for real-time and continuous monitoring of metal particle emission. Micromachines (Basel), 2021, 12: 825

[15]Park J, Yoo S-J, Yoon J-s, et al. Inductive particle detection system for real-time monitoring of metals in airborne particles. Sensors and Actuators A: Physical, 2021, 332: 113153.

[16]Wang L Y, Zhong H, Li L, et al. Influence of coil spacing on output signal of oil abrasive particle detection sensor. Lubrication Engineering, 2020, 45: 69-75

[17]Wang X Y, Chen P, Luo J F, et al. Characteristics and superposition regularity of aliasing signal of an inductive debris sensor based on a high-gradient magnetic field. IEEE Sensors Journal, 2020, 20: 10071-10078

[18]Bai C Z, Zhang H P, Wang W Q, et al. Inductive-capacitive dual-mode oil detection sensor based on magnetic nanoparticle material. IEEE Sensors Journal, 2020, 20: 12274-12281
[19]Shi H, Zhang H P, Ma L H, et al. An impedance debris sensor based on a high-gradient magnetic field for high sensitivity and high throughput. IEEE Transactions on Industrial Electronics, 2021, 68: 5376-5384

[20]Chen H, Wang L Y, and Chen T. Influence of coil parameters on magnetic field uniformity of inductance abrasive sensor. Journal of Electronic Measurement and Instrumentation, 2020, 34: 10-16

[21]Zhang H P, Zeng L, Teng H B, et al. A novel on-chip impedance sensor for the detection of particle contamination in hydraulic oil. Micromachines (Basel), 2017, 8: 249.

[22]Bai C Z, Zhang H P, Zeng L, et al. Inductive magnetic nanoparticle sensor based on microfluidic chip oil detection technology. Micromachines (Basel), 2020, 11:183-196

[23]Shi H T, Zhang H P, Gu C Z, et al. A multi-parameter on-chip impedance sensor for the detection of particle contamination in hydraulic oil. Sensors and Actuators A: Physical, 2019, 293: 150-159

[24]Du L, Zhu X L, Han Y, et al. Improving sensitivity of an inductive pulse sensor for detection of metallic wear debris in lubricants using parallel lc resonance method. Measurement Science and Technology, 2013, 24: 75106-75117

[25]Ren Y J, Zhao G F, Qian M, et al. A highly sensitive triple-coil inductive debris sensor based on an effective unbalance compensation circuit. Measurement Science and Technology, 2018, 30: 015108

[26]Luo J S, Yu D J, and Liang M. Enhancement of oil particle sensor capability via resonance-based signal decomposition and fractional calculus. Measurement, 2015, 76: 240-254

[27]Hong W, Wang S P, Liu H K, et al. A hybrid method based on band pass filter and correlation algorithm to improve debris sensor capacity. Mechanical Systems and Signal Processing, 2017, 82: 1-12

[28]Liu H K, Li T Y, Hong W, et al. Using multi-window correlation to improve sensitivity and adaptability for oil debris detections. Measurement, 2021, 176: 109236.

[29]Zhu X L, Du L, and Zhe J. A $3 \times 3$ wear debris sensor array for real time lubricant oil conditioning monitoring using synchronized sampling. Mechanical Systems and Signal Processing, 2017, 83: 296-304

[30]Wu S, Liu Z J, Yu K Z, et al. A novel multichannel inductive wear debris sensor based on time division multiplexing. IEEE Sensors Journal, 2021, 21: 11131-11139

[31]Feng S, Yang L L, Qiu G, et al. An inductive debris sensor based on a high-gradient magnetic field. IEEE Sensors Journal, 2019, 19: 2879-2886

[32]Xiao H, Wang X Y, Li H C, et al. An inductive debris sensor for a large-diameter lubricating oil circuit based on a high-gradient magnetic field. Applied Sciences, 2019, 9: 1546.

[33]Xiao H, Zhou W, Luo J F, et al. An inductive sensor based on the high-gradient static magnetic field for full flow debris monitoring. Chinese Journal of Scientific Instrument, 2020, 41: 10-17

[34]Ren Y J, Li W, Zhao G F, et al. Inductive debris sensor using one energizing coil with multiple sensing coils for sensitivity improvement and high throughput. Tribology International, 2018, 128: $96-103$

\section{Biographical notes}

Kai Li, born in 1979, is currently an associate professor at School of Information and Communication Engineering, North University of China, China. His research interests include 
man-machine system and mechanical fault diagnosis.

Tel: +86-13753156898; E-mail: likai@nuc.edu.cn

WenBin Bai, born in 1997, is currently a master candidate at State Key Laboratory of Fluid Power Transmission and Control, Zhejiang University, China.

E-mail: s1905009@st.nuc.edu.cn

Yuan Li, born in 1982, is currently an associate professor at School of Information and Communication Engineering, North University of China, China.

E-mail: liyuan82@nuc.edu.cn

HaoSen Dong, born in 1998, is currently a master candidate at State Key Laboratory of Fluid Power Transmission and Control, Zhejiang University, China.
E-mail: sz202105050@st.nuc.edu.cn

RuiQing Bian, born in 1998, is currently a master candidate at State Key Laboratory of Fluid Power Transmission and Control, Zhejiang University, China.

E-mail: sz202105017@st.nuc.edu.cn

QingQing Gong, born in 1996, is currently a master candidate at State Key Laboratory of Fluid Power Transmission and Control, Zhejiang University, China.

E-mail: s1905076@st.nuc.edu.cn

YaJun Ren, born in 1996, is currently a master candidate at State Key Laboratory of Fluid Power Transmission and Control, Zhejiang University, China.

E-mail: s1905036@st.nuc.edu.cn 